Journal of Applied Pharmaceutical Science Vol. 6 (01), pp. 007-014, January, 2016

Available online at http://www.japsonline.com

DOI: $10.7324 / \mathrm{JAPS} .2016 .600102$

ISSN 2231-3354 (cc)) BY-NC-SA

\title{
Spectrophotometric Determination of Gentamicin Loaded PLGA Microparticles and Method Validation via Ninhydrin-Gentamicin Complex as a Rapid Quantification Approach
}

\author{
Ahmad Fahmi Harun Ismail ${ }^{1}$, Farahidah Mohamed ${ }^{1 *}$, Luqman Muizzuddin Mohd Rosli ${ }^{1}$, Mohd Affendi Mohd Shafri ${ }^{2}$, \\ Muhammad Salahuddin Haris ${ }^{1}$, Anugerah Budipratama Adina ${ }^{2}$ \\ ${ }^{1}$ Kulliyyah of Pharmacy (KOP), International Islamic University Malaysia (IIUM), Jalan Sultan Ahmad Shah, Bandar Indera Mahkota, 25200 Kuantan, \\ Pahang Darul Makmur, Malaysia. ${ }^{2}$ Kulliyyah of Allied Health Sciences (KAHS), International Islamic University Malaysia (IIUM), Jalan Sultan Ahmad \\ Shah, Bandar Indera Mahkota, 25200 Kuantan, Pahang Darul Makmur, Malaysia.
}

\begin{tabular}{l} 
ARTICLE INFO \\
\hline Article history: \\
Received on: 05/11/2015 \\
Revised on: 09/12/2015 \\
Accepted on: 22/12/2015 \\
Available online: 26/01/2016 \\
\hline Key words: \\
Spectrophotometry; \\
Gentamicin; Ninhydrin; \\
Microparticles; poly(lactic- \\
co-glycolic acid).
\end{tabular}

\begin{abstract}
The purpose of this study was to provide a quantification method with rapid, sensitive, reproducible and cost effective for gentamicin in the form of ninhydrin-gentamicin complex. The utilization of spectrophotometric module to validate the method development for gentamicin loaded microparticles was intended to provide alternative quantification method without undermining the sensitivity of the developed method. The microparticles fabrication process was proven to be suitable in encapsulating gentamicin by using poly(lactic-coglycolic acid) PLGA without compromising the efficacy of the antibiotic itself. The linearity of 6 known concentrations of ninhydrin-gentamicin complex was obtained with the $\mathrm{R}^{2}$ of 0.9998 . The interday and intraday precisions were determined with the acceptance \%RSD values of less than $2 \%$. The highest \%RSD value was $1.09 \%$ which suggested the method to be acceptably precise. The limit of detection (LOD) and limit of quantification (LOQ) values were recorded to be at 0.016 and $0.196 \mathrm{mg} / \mathrm{mL}$ respectively. The \% recovery of 4 known concentrations indicated the accuracy of the method was high with the recovery range between $98.66 \%$ and $101.8 \%$. The parameters analyzed in this study were in accordance with ICH Q2 (R1) guidelines. This quantification method was a promising approach to provide a rapid and cost effective in evaluating gentamicin concentration for in-vitro applications.
\end{abstract}

\section{INTRODUCTION}

Gentamicin is an antibiotic belongs to the family of aminoglycosides that was discovered in 1963 and was isolated from Micromonospora purpurea. It is readily soluble in water due to the presence of hydroxyl groups on its chemical structure, but practically insoluble in organic solvents (Šoltés, 1999). The narrow therapeutic range possesses by gentamicin has made

\footnotetext{
* Corresponding Author

Farahidah Mohamed, Kulliyyah of Pharmacy (KOP), International Islamic University Malaysia (IIUM), Jalan Sultan Ahmad Shah, Bandar Indera Mahkota, 25200 Kuantan, Pahang Darul Makmur, Malaysia. Tel: +6095716400 Fax:+6095716775, Email: farahidah@iium.edu.my
}

the antibiotic to be one of the heated debate topics amongst the physicians, whether it should not be used at all or merely that it should not be used extensively (Ariano et al., 2008). Ototoxicity and nephrotoxicity have been reported widely to be the main concern whenever the aminoglycosides are administered to the patients (Šoltés, 1999; Ariano et al., 2008; Al-Hamad, 2014; Yusof et al., 2014). This concern has become the driving factor for many researchers to develop a method that can minimize the bioavailability of aminoglycoside systemically but at the same time maximize the therapeutic effect only where it is needed. A carrier system with sustained release characteristic is the common option available to overcome the problem (Chung and Huang, 2001; Balmayor et al., 2012; Abed and Couvreur, 2014). 
There is a quite considerable amount of literature has been published pertaining the sustained release of gentamicin using biodegradable polymers such as poly(lactic-co-glycolic acid) or commonly known as PLGA (Friess and Schlapp, 2002; Schlapp and Friess, 2003; Ismail et al., 2012). The quantification method was developed and validated based on the requirements listed by The International Conference on Harmonization of Technical Requirements for Registration of Pharmaceuticals for Human Use (ICH). Although gentamicin has been extensively used as the subject for sustained release formulations, literature reviews have indicated that there is no general agreement about developing an analytical method validation (AMV) for gentamicin by using UVspectrophotometer. It is a rapid and cost effective with significantly moderate sensitivity in detecting gentamicin using UV-spectrophotometry as compared to analytical methods using HPLC or GC-MS.

When it comes to the relation between transmittance and absorbance of a solution in the application of UVspectrophotometry detection, Beer-Lambert Law is one of the basic principles in understanding the concept. Briefly, the strength of one light beam passes through a translucent cell or cuvette holding an absorbing solution will have an inversely proportion between the intensity of the beam detected and the concentration of the solution (Behera et al., 2012; Ismail et al., 2015). It was reported elsewhere that the concentration of gentamicin was determined by using fluorescent assay at the wavelength of 450 $\mathrm{nm}$, with the $\mathrm{R}^{2}$ value for the linearity constructed at this wavelength was less than 0.98 (Lecároz et al., 2006). Additionally, the same literature is also reported that the correlation for linearity constructed using HPLC for gentamicin extracted from microparticles using dichloromethane and sodium hydroxide gives the values of 0.889 and 0.855 respectively.

The aim of this study is to develop a method to quantify the presence of gentamicin in the form of gentamicin-ninhydrin complex by using UV-spectrometer. The method would be developed and validated based on the requirements listed under ICH Q2 (R1) guidelines. In addition, the study was done to prove the stability and the efficacy of the gentamicin for post fabrication process by evaluating the response of the antibiotic against staphylococcus aureus.

It is believed that the analytical method developed and validated in this study would provide a rapid and cost effective in vitro quantification for gentamicin.

\section{MATERIALS}

\section{Chemicals and Reagent}

Gentamicin sulphate was purchased from local pharmacy. Purasorb® PLGA with three different intrinsic viscosities; $0.2 \mathrm{dL} / \mathrm{g}(14 \mathrm{kDa}), 0.4 \mathrm{dL} / \mathrm{g}(34 \mathrm{kDa})$ and $1.0 \mathrm{dL} / \mathrm{g}$ (100 kDa) were purchased from Purac (Netherland). Ninhydrin with laboratory reagent grade was purchased from Fisher Scientific (United Kingdom). Poly Vinyl Alcohol (PVA) with molecular weight approximately $115 \mathrm{kDa}$ (BDH Laboratory
Supplies, England) was used. All solvents were purchased from Merck (Germany) with analytical grade. Staphylococcus aureus (ATCC 29523) was purchased from Microbiologics ${ }^{\circledR}$ (USA).

\section{Instrumentation}

The analysis was done using single beam UVspectrophotometry module (U-1900 Toshiba, Japan) with wavelength scanned from $600 \mathrm{~nm}$ to $190 \mathrm{~nm}$. The silica quartz square cell cuvette (Optima, Japan) with transmittance of $51.4 \pm$ $0.3 \%$ at wavelength $545 \mathrm{~nm}$ was used throughout the whole experiment.

\section{EXPERIMENTAL METHODS}

\section{Colorimetric Reagent}

Accurately $50 \mathrm{mg}$ of ninhydrin powder was weighted and dissolved with $10 \mathrm{~mL}$ of phosphate buffered saline (PBS) solution $(\mathrm{pH} 7.4)$ to get the stock solution with the concentration of 5 $\mathrm{mg} / \mathrm{mL}$. The stock solution was kept under $4{ }^{\circ} \mathrm{C}$ and was protected from any light source.

\section{Fabrication of Gentamicin Microparticles}

Modified double emulsion solvent evaporation method was adopted from Ismail et al. (2012). Briefly, PLGA was dissolved in $2 \mathrm{~mL}$ of chloroform before mixed up with $200 \mu \mathrm{L}$ aqueous solution containing $100 \mathrm{mg}$ of gentamicin to be regarded as the primary emulsion. Secondary emulsion was achieved by mixing between primary emulsion $8 \mathrm{~mL}$ of $1 \% \mathrm{w} / \mathrm{v}$ PVA solution, before it was transferred into the hardening tank $(100 \mathrm{~mL}$ of $1 \%$ w/v PVA solution) for solvent evaporation process which took 3 hours. The mixing process for primary and secondary emulsions was done by using ultra sonicator (QSonica, USA; 3 cycles for 3 seconds each at $50 \mathrm{MHz}$ ). The microparticles were collected by mean of centrifugation (4000 rpm, $5 \mathrm{~min}$ ) and followed by washing to remove PVA before they were lyophilized under reduced pressure.

Table 1: The nine formulations of the microparticles based on the ratio and the blends of PLGA used. The amount of gentamicin loaded for each formulation was $100 \mathrm{mg}$ each.

\begin{tabular}{ccc}
\hline Formulation & Intrinsic Viscosity $(\mathbf{d L} / \mathbf{g})$ & PLGA ratio \\
\hline F1 & $0.2 / 0.4$ & $50: 50$ \\
F2 & $0.2 / 1.0$ & $50: 50$ \\
F3 & $0.4 / 1.0$ & $50: 50$ \\
F4 & $0.2 / 0.4$ & $75: 25$ \\
F5 & $0.2 / 0.4$ & $25: 75$ \\
F6 & $0.2 / 1.0$ & $75: 25$ \\
F7 & $0.2 / 1.0$ & $25: 75$ \\
F8 & $0.4 / 1.0$ & $75: 25$ \\
F9 & $0.4 / 1.0$ & $25: 75$ \\
\hline
\end{tabular}

The gentamicin loaded microparticles were evaluated based on the particle size distribution and the amount of drug entrapment. For this study, nine different formulations were selected based on the combinations of the PLGA molecular weights and the ratio used between those molecular weight blends (Table 1). 


\section{Particle Size Distribution}

The particle size distribution was evaluated by using Laser Particle Size Analyzer (Bettersize Instruments, China) and was done in triplicate. The particles were suspended in deionised water before subjected for measurement.

\section{Encapsulation Efficiency}

Briefly, 5-8 mg of gentamicin loaded microparticles were placed in a tube and re-dissolved using $1 \mathrm{~mL}$ of chloroform followed with the addition of another $1 \mathrm{~mL}$ of PBS solution $(\mathrm{pH}$ 7.4).

This method was adopted from Ismail et al. (2012) and modified accordingly. The tube was rotated end-to-end for 1 hour before $800 \mu \mathrm{L}$ of the aqueous part was extracted and transferred into another tube after centrifugation process (4000 rpm, $5 \mathrm{~min}$ ). Equal amount of colorimetric reagent was added to the solution before it was subjected to heat treatment using water bath $\left(95{ }^{\circ} \mathrm{C}\right.$, $15 \mathrm{~min}$ ) and followed with cold treatment using ice cold water bath for 10 minutes. This final solution was subjected to UVspectrophotometry evaluation with the wavelength of $418 \mathrm{~nm}$. The quantification for each formulation was done in triplicate and the average values were recorded.

\section{Gentamicin Efficacy for Post Microparticle Fabrication}

In short, $10 \mathrm{mg}$ of gentamicin loaded microparticles were dissolved in $1 \mathrm{~mL}$ chloroform and another $1 \mathrm{~mL}$ of PBS solution was added. End-to-end rotation was done for 1 hour to ensure all the microparticles were dissolved before the aqueous part was extracted. $100 \mu \mathrm{L}$ from the extracted solution was dropped on a blank paper disk and tested against staphylococcus aureus. The disk diffusion method adopted and modified from Ismail et al. (2012) was utilized by preparing the lawn of S. aureus by using nutrient agar. Optical density of the bacteria broth was measured using UV-spectrometer (Hitachi U1900 Single Beam, Tokyo Japan) which corresponded to $0.5 \mathrm{McFarland}$ standard. The concentration of $10^{8}$ colony forming unit $(\mathrm{CFU}) / \mathrm{mL} \quad(0.5$ McFarland Standard) was achieved by measuring the optical density which was between $0.008-0.1$ absorbance value (Andrews, 2001).

The $S$. aureus was cultured by using nutrient broth and the nutrient agar was used as the medium for the bacteria lawn. The zone of inhibition for each formulation of microparticles was measured by using Absolute Digimatic Caliper Mitutoyo (Japan) with sensitivity up to $0.01 \mathrm{~mm}$.

\section{METHOD DEVELOPMENT AND VALIDATION}

\section{Preparation of Gentamicin Stock Solution}

Accurately $1 \mathrm{~g}$ of gentamicin powder was weighted and dissolved with $10 \mathrm{~mL}$ of PBS solution (pH of 7.4) and stored at 4 ${ }^{\circ} \mathrm{C}$. This stock solution with final concentration of $100 \mathrm{mg} / \mathrm{ml}$ $(10 \% \mathrm{w} / \mathrm{v})$ was diluted accordingly whenever required. The PVA $1 \% \mathrm{w} / \mathrm{v}$ was prepared by dissolving the PVA powder by using ultra pure water.

\section{Specificity}

Gentamicin-ninhydrin complex in PBS would give a purple colour with clear non turbid solution. Gentamicin alone in aqueous solution would not give any colour at relatively low concentration and could not be detected with UV spectrophotometer. Initially, a wavelength scan was performed to get the best peak for gentamicin-ninhydrin complex without any possible interference with other possible components in the solution. PBS solution was used as blank solution and was regarded as background reading. The same scanning process was done for PLGA and ninhydrin alone to eliminate any false positive in selecting the best peak for gentamicin-ninhydrin complex. The determined wavelength would be used throughout the study whenever needed.

\section{Linearity and Standard Curve}

The linearity of the six different known concentrations of gentamicin-ninhydrin complex was subjected to the previously determined wavelength. The absorbance of the respected concentrations was recorded. A concentration vs. absorbance graph was plotted on which the equation $y=m x+c$ and the $\mathrm{R}^{2}$ were established. The concentration of the analyte was determined with the notation of $x$ with $y$ is the absorbance value. The correlation coefficient $\mathrm{R}^{2}$ value for all the standard curves constructed for this study was set to be not less than 0.995 as the one of the acceptance criteria (Rajesh et al., 2014; Ismail et al., 2015).

\section{Accuracy and Precision}

For the accuracy, four known different concentrations of gentamicin-ninhydrin complex were subjected to the established wavelength $(418 \mathrm{~nm})$ and the experimental concentrations were recorded. Each concentration was subjected to the wavelength in triplicate and the mean and standard deviation were recorded. The precision was evaluated by taking into consideration the percentage deviation of the absorbance values obtained from the actual concentrations. The interday precision was carried out by doing the measurement in 3 different days for 3 different preparations. The intraday precision on the other hand was done by subjecting four known sample concentrations to the same wavelength $(418 \mathrm{~nm})$ but in 3 different specific time points on the same day. The percentage recovery should not be less than $98 \%$ and more than $102 \%$ with \% RSD value is smaller than $2.0 \%$ to meet the acceptance criteria (Rajitha et al., 2011; Ismail et al., 2015).

\section{Limit of Detection (LOD) and Limit of Quantification (LOQ)}

The evaluation for both LOD and LOQ was done by subjecting the blank solution (PBS solution with $\mathrm{pH}$ of 7.4) to the same wavelength used to quantify the samples. There are specific calculations to determine both LOD and LOQ as described below;

$$
\mathrm{LOD}=\frac{3.3 \times S . D}{m} \quad \mathrm{LOQ}=\frac{10 \times S . D}{m}
$$


S.D or standard deviation in the formula was referring to the standard deviation of the absorbance values of the blank and $m$ is the slope of the standard curve constructed previously (Iqbal et al., 2013). All readings for LOD and LOQ were taken 3 times.

\section{Robustness}

The robustness of the method was determined by intentionally introducing small changes in the procedures or sample preparations. The deviations of data collected for robustness were compared to the original data and any significant changes were recorded. The wavelength was intentionally changed to $421 \mathrm{~nm}, 415 \mathrm{~nm}$ and $400 \mathrm{~nm}$ and the standard curves for each wavelength were constructed with the correlation coefficient $\mathrm{R}^{2}$ values were recorded. In addition, the second analyst was assigned to construct the standard curve to get the $\mathrm{R}^{2}$ values and the linear equations based on the absorbance values of six different known concentrations of gentamicin-ninhydrin complex. The method was considered to be robust if the $\mathrm{R}^{2}$ values obtained were not less than 0.995 .

\section{RESULT AND DISCUSSION}

\section{Particle Size Distribution and Encapsulation Efficiency}

The size distribution for all formulations indicate that the size of the particles fell in the range between $1.624 \mu \mathrm{m} \pm 0.194$ to $5.103 \mu \mathrm{m} \pm 1.537$ (Table 2). Relatively, the size distribution of the microparticles for all formulations could be considered as homogenously dispersed. This homogenicity was determined based on the span values for each of the formulations. The span value was calculated based on the size distributions of the particles at 3 different distributions (10\%, 50\% and 90\%) with the formulation as described as follow:

$$
\text { Span value }=\frac{D 90-D 10}{D 50}
$$

Whereby D90, D10 and D50 are the particle distribution at $90 \%, 10 \%$ and $50 \%$ respectively. The span value lower than 1 indicates that the size distribution can be considered as homogenously dispersed. Although the particle size was homogenously distributed, the size distribution was not really a top priority in this study since the microparticles were intended to be used locally at the site of infection without having to go through any parenteral routes. The results of the encapsulated efficiency (EE) indicate that the molecular weight and the PLGA blends influenced the amount of gentamicin encapsulated using this fabrication method. Double emulsion solvent evaporation method is meant to encapsulate drugs which are hydrophilic in nature such as gentamicin (Ismail et al., 2012). Figure 1 showed that although gentamicin was able to be encapsulated within the microparticles, the highest amount of gentamicin encapsulated was $42.98 \% \pm 2.60$ for F8. The lowest $\mathrm{EE}(11.64 \% \pm 2.66)$ however represented by the formulation (F1) employed $0.2 \mathrm{dL} / \mathrm{g}$ and $0.4 \mathrm{dL} / \mathrm{g}$ of PLGA with the ratio of 50:50. Among the plausible explanations for this finding is that the molecular weight of the PLGA plays a vital role in determining the amount of gentamicin encapsulated in the microparticles. It was obvious that higher molecular weight (higher intrinsic viscosity) with higher ratio would trap more gentamicin as compared to the lower molecular weight of PLGA.

Although the contradiction was observed, this finding was recorded with a fabrication system employed the single polymer emulsion and mechanical shearing force. The current study however had utilized the ultrasonic wave as the mean of homogenizing the emulsion in addition to the employment of molecular weight blends of the polymer used. The emulsion is more stable when the polymer used is high in molecular weight. Thicker emulsion will be formed with high molecular weight polymer as compared to low molecular weight polymer. Hence, the droplets formed are more stable with less coalescence rate between the droplets.

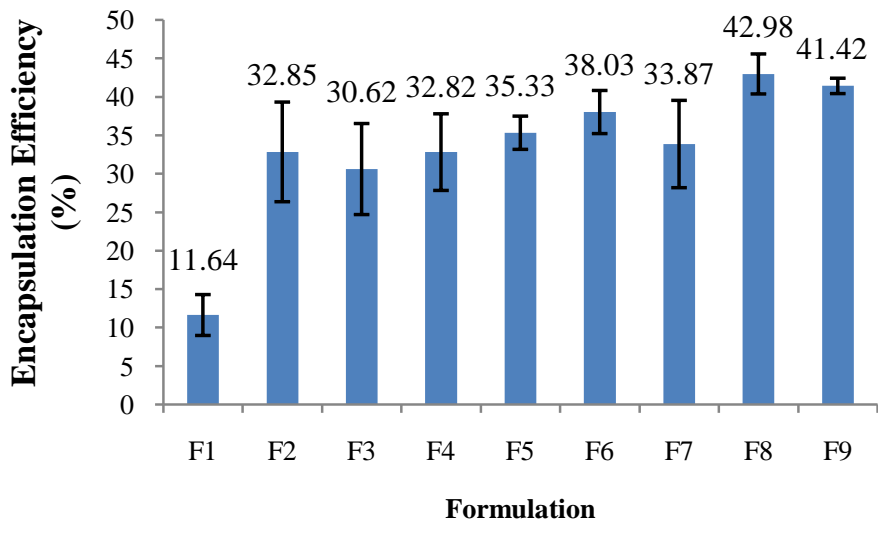

Fig. 1: Encapsulation efficiency of the gentamicin loaded microparticles based on the formulations employed.

Table 2: The size distribution of the gentamicin loaded microparticles according to the formulations.

\begin{tabular}{cccc}
\hline Formulation & $\begin{array}{c}\text { Size Distribution }(\boldsymbol{\mu m}) \\
(\mathbf{n}=\mathbf{3})\end{array}$ & S.D $( \pm \boldsymbol{\mu m})$ & $\begin{array}{c}\text { Span } \\
\text { Value }\end{array}$ \\
\hline F1 & 4.973 & 0.427 & 0.282 \\
F2 & 2.700 & 0.394 & 0.299 \\
F3 & 2.346 & 0.246 & 0.400 \\
F4 & 5.103 & 1.537 & 0.239 \\
F5 & 2.973 & 0.127 & 0.353 \\
F6 & 2.932 & 0.432 & 0.292 \\
F7 & 1.624 & 0.194 & 0.426 \\
F8 & 1.785 & 0.369 & 0.430 \\
F9 & 3.244 & 0.792 & 0.305 \\
\hline
\end{tabular}

\section{Gentamicin Efficacy for Post Microparticle Fabrication}

The fabrication process to encapsulate gentamicin proved to be suitable and compatible without changing the efficacy of drug itself. The efficacy for post fabrication process was evaluated by extracting the encapsulated gentamicin and subjecting to the bacteria lawn. Figure 2 showing the antimicrobial action of gentamicin against the $S$. aureus. It was found that the efficacy of gentamicin was still intact based on the formation of zones of inhibition. Although the diameter of the zones of inhibition could be measured according to the formulations used, it was not scientifically suitable to represent the data to correspond the amount of drug loading unless all the variables were strictly controlled. Interestingly based on the result obtained, it was 
proven that the encapsulated gentamicin could still be effective against the $S$. aureus. The diameters of the zone of inhibition according to the formulation were tabulated under Table 3 . Gentamicin has the ability to bind to a conserved sequence of rRNA of the bacteria which is very close to the site of codonanticodon recognition of the 30S subunits (Yoshizawa et al., 1998).

This binding would reduce the accuracy of the bacteria in translating the genetic material and inhibit the translocation of the ribosomes which interrupting the synthesis of protein materials. This polycationic inhibitor of the $30 \mathrm{~S}$ ribosomal subunit would cause the bactericidal against $S$. aureus by disrupting the cell membrane transportation and the permeability of the cell wall (McLawhon, 2012).

Table 3: The average diameters of zone of inhibition according to the formulations.

\begin{tabular}{ccc}
\hline Formulation & $\begin{array}{c}\text { Average Diameter } \\
(\mathbf{m m})\end{array}$ & $\begin{array}{c}\text { Standard Deviation } \\
(\mathbf{\pm} \mathbf{m m})\end{array}$ \\
\hline F1 & 24.78 & 1.36 \\
F2 & 23.22 & 2.88 \\
F3 & 22.37 & 0.39 \\
F4 & 25.60 & 1.80 \\
F5 & 25.02 & 1.88 \\
F6 & 25.82 & 0.91 \\
F7 & 25.03 & 0.62 \\
F8 & 22.30 & 1.68 \\
F9 & 24.20 & 2.75 \\
\hline
\end{tabular}
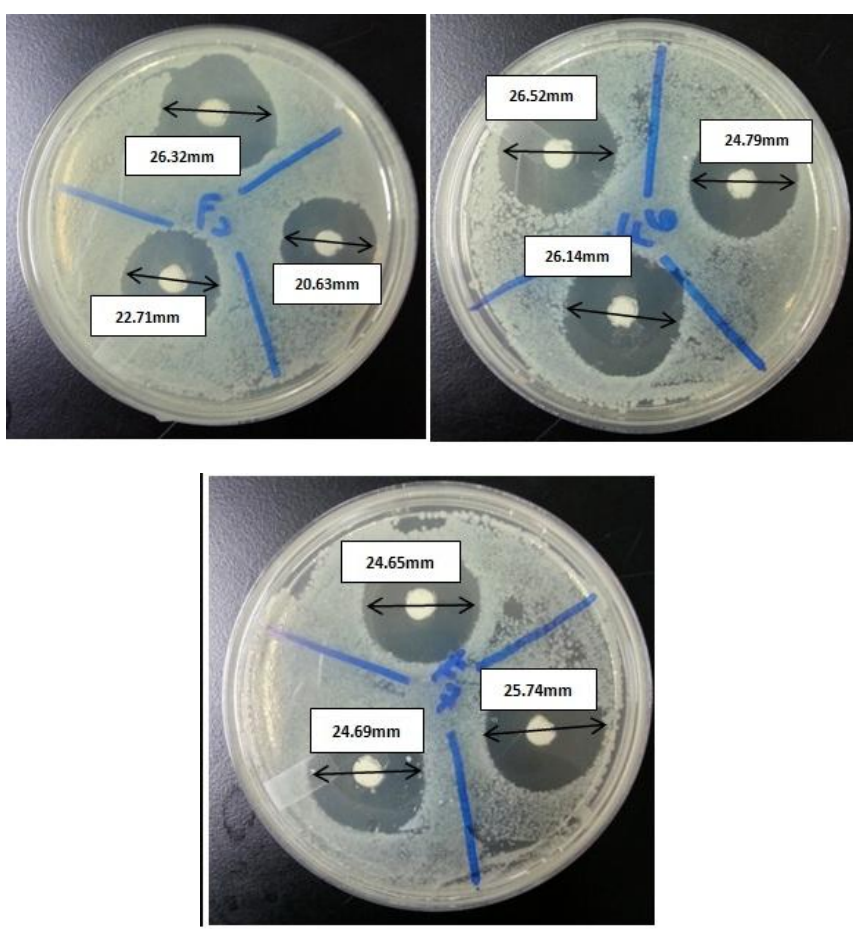

Fig. 2: The zone of inhibition for some of the formulations (F2, F6 and F7) gentamicin against Staphylococcus aureus.

\section{Specificity}

In this study, gentamicin loaded microparticles were fabricated using PLGA and the amount of drug loading for post fabrication was characterized by using UV-spectrophotometer. Since gentamicin is poorly absorbed by the visible light and UVlight, an indirect spectrophotometry method needed to be developed.

Ninhydrin was used to produce a colorimetric procedure in order to quantify the gentamicin encapsulated. The principle reaction between ninhydrin and gentamicin is based on the chemical interaction of ninhydrin with the primary and secondary amine groups present in the gentamicin chemical structure which produces purple colour (Frutos et al., 2000). Spectrophotometric method with rapid, sensitive and cost effective for analysis of a single component in solution can be achieved for any drugs that obey the Beer-Lambert Law.

Based on the wavelength scanning done for the gentamicin-ninhydrin complex, the wavelength of $418 \mathrm{~nm}$ was selected due to the colorimetric appearance of the solution (clear purple) which indicated the detection should not be under UV region (below $400 \mathrm{~nm}$ ). In addition, the scanning spectrum from $600 \mathrm{~nm}$ to $200 \mathrm{~nm}$ verified that at $418 \mathrm{~nm}$ region, the detection would not be interrupted with the wavelength of PLGA and ninhydrin alone itself (Figure 3).

Although the $418 \mathrm{~nm}$ wavelength was determined by the UV module, the gentamicin-ninhydrin complex was also subjected to $400 \mathrm{~nm}$ to evaluate the degree of deviation by constructing the linearity and obtaining the correlation coefficient $\mathrm{R}^{2}$ value. Based on the $\mathrm{R}^{2}$ value obtained from the constructed linearity at $400 \mathrm{~nm}$, it was below the acceptance requirement $(0.9772)$ which is not less than 0.995 . This undesired correlation coefficient value had made the wavelength at $418 \mathrm{~nm}$ as the best choice for the specificity in detecting gentamicin-ninhydrin complex.

\section{Linearity and Standard Curve}

The linearity was determined based on the constant direct proportionate relation between the concentration of gentamicinninhydrin complex and the absorbance values specifically at 418 nm wavelength.

Six known concentrations of gentamicin-ninhydrin complex were prepared $(2.0,4.0,5.0,6.0,8.0$ and $10.0 \mathrm{mg} / \mathrm{mL})$ and subjected to $418 \mathrm{~nm}$ to get the absorbance values. The standard curve obtained from the UV-spectrophotometry based on these 6 concentrations was in linear form with the equation and the $\mathrm{R}^{2}$ of $y=0.069 x+0.007$ and 0.9998 respectively. The standard curve is illustrated in Figure 4.

The readings for each concentration were done in triplicate and the average of those three was calculated to maximize data reliability. The inconsistency of the readings from the UV module could be neglected since the background solvent used in this study was PBS solution. It was mentioned by Ismail and co-workers that volatile organic solvent such as dichloromethane used as the background solution would contribute to the inconsistency of the readings obtained due to the rapid evaporation of the solvent itself (Ismail et al., 2015). 


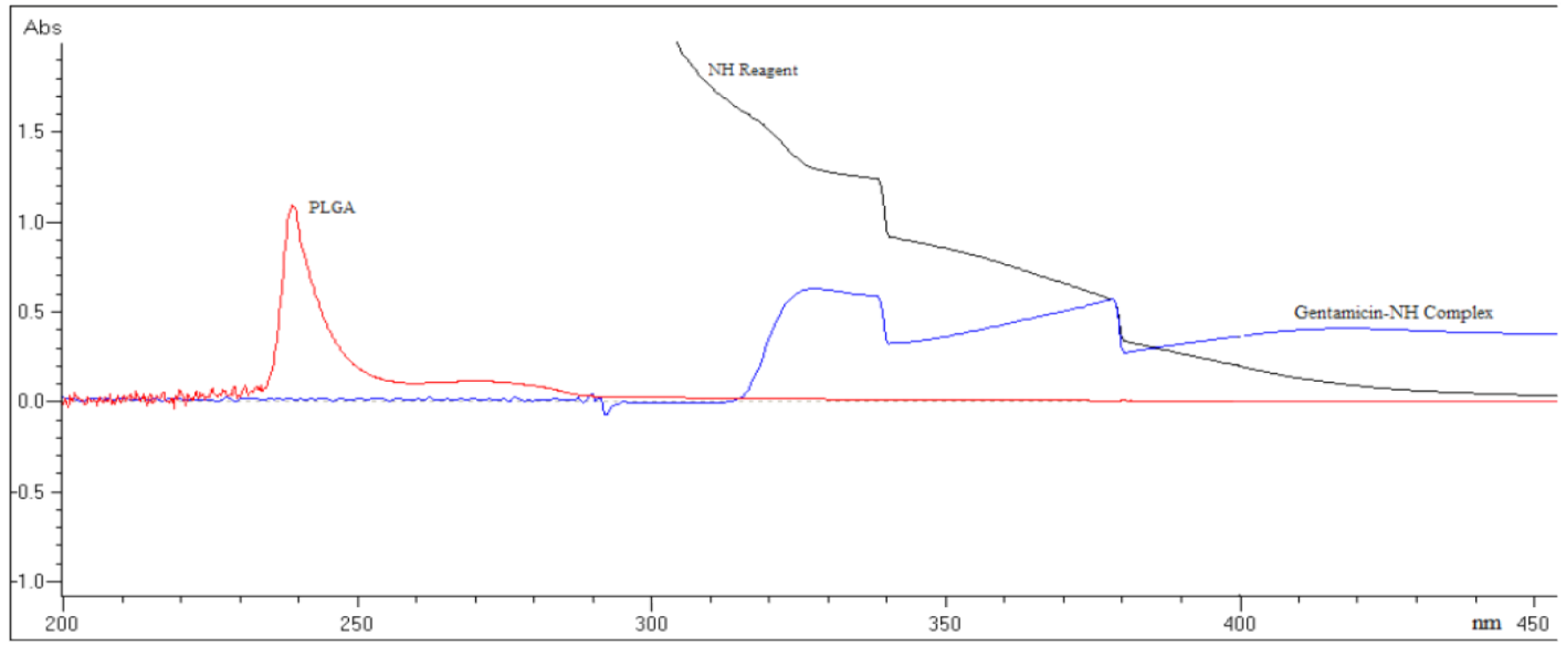

Fig. 3: The wavelength scanning spectrum of PLGA, ninhydrin (NH) reagent, and gentamicin-ninhydrin complex for specificity.

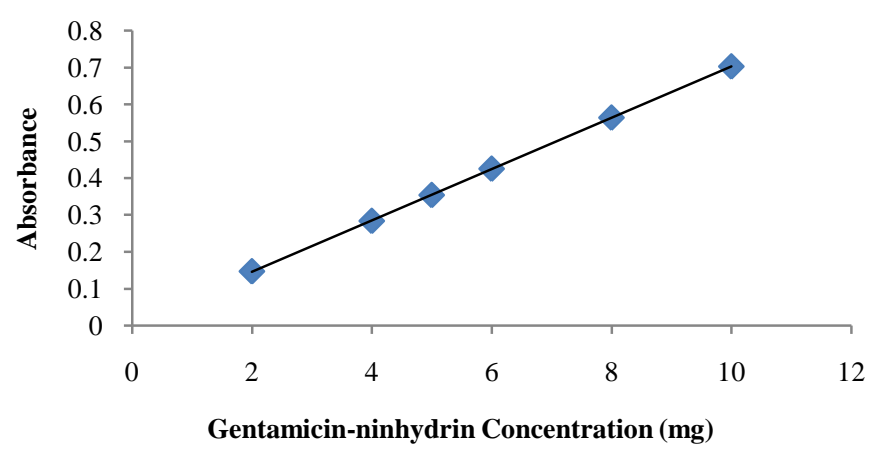

Fig. 4: The standard curve constructed for linearity study.

\section{Accuracy and Precision}

The intermediate precision was carried out by using 4 known concentrations of the gentamicin-ninhydrin complex (2.0, 4.0, 6.0 and $8.0 \mathrm{mg} / \mathrm{mL}$ ) and was measured in 3 consecutive days. The 4 known concentrations were freshly prepared each day and for each concentrations, the readings were taken in triplicate. Based on the data for intermediate precision (Table 4), the quantification method was considered to be precise in detecting gentamicin-ninhydrin complex at different concentrations as the \%RSD obtained was less than $2.0 \%$. This similar finding was observed and reported by a few others with the same acceptance criteria monitored (Behera et al., 2012; Ismail et al., 2015).

For repeatability study (intraday precision), similar outcome was observed where 3 subsequence readings were taken on the same day. All 4 known concentrations (2.0, 4.0, 6.0 and 8.0 $\mathrm{mg} / \mathrm{mL}$ ) were freshly prepared each time. The repeatability data was tabulated in Table 5 with the \%RSD for all concentrations shown the values less than $2.0 \%$. This quantification method was considered to be precise and met the requirement set by ICH Q2 (R1) guidelines. The accuracy study for this method was modified due to the high intensity of purple color if the concentration of gentamicin-ninhydrin complex was raised up to $100 \% \mathrm{w} / \mathrm{v}$ and more.

The absorbance reading would jump over 2.0 when the transparency of the solution dropped drastically. The detection of the module for such over concentrated sample would drop below $0.1 \%$ for the transmittance.

Low transmittance resulted from too concentrated sample would give a considerably high standard error in the reading. Consequently, the same concentrations were used $(2.0,4.0,6.0$ and $8.0 \mathrm{mg} / \mathrm{mL}$ ) in determining the accuracy of the method without compromising the requirement of acceptance of the data collected.

Almost the same issue was described in another study done elsewhere with the quantification method developed for Nigella sativa oil using UV spectrophotometry (Ismail et al., 2015). Table 6 indicates the accuracy data collected with each concentration was done in triplicate. The average recovery was in between the acceptance range ( $98 \%$ to $102 \%$ ) with the $\%$ RSD was less than $2.0 \%$.

\section{Limit of Detection (LOD) and Limit of Quantification (LOQ)}

The LOD and LOQ were calculated based on the formulations mentioned under the experimental methods. By considering the control experimental condition for this study, the LOD and LOQ values were $0.016 \mathrm{mg} / \mathrm{mL} \pm 0.0003 \mathrm{mg} / \mathrm{mL}$ and $0.196 \mathrm{mg} / \mathrm{mL} \pm 0.001 \mathrm{mg} / \mathrm{mL}$ respectively with the slope of the standard curve was 0.07 . The correlation coefficient $\mathrm{R}^{2}$ for the standard curve was 0.9998 . 
Table 4: The intermediate precision was obtained by using UV-spectrophotometry for gentamicin-ninhydrin complex.

\begin{tabular}{|c|c|c|c|c|}
\hline Concentration $(\mathrm{mg} / \mathrm{mL})$ & Average Actual Concentration $(\mathrm{mg} / \mathrm{mL})(\mathrm{n}=3)$ & S.D & $\mathbf{R}^{2}$ For Standard Curve & Precision (\%RSD) \\
\hline Day 1 & & & 0.9998 & \\
\hline 2.000 & 2.031 & 0.003 & & 0.159 \\
\hline 4.000 & 4.103 & 0.003 & & 0.068 \\
\hline 6.000 & 6.054 & 0.007 & & 0.108 \\
\hline 8.000 & 8.058 & 0.015 & & 0.183 \\
\hline Day 2 & & & 0.9995 & \\
\hline 2.000 & 2.039 & 0.001 & & 0.035 \\
\hline 4.000 & 4.055 & 0.001 & & 0.008 \\
\hline 6.000 & 6.055 & 0.001 & & 0.007 \\
\hline 8.000 & 8.054 & 0.001 & & 0.012 \\
\hline Day 3 & & & 0.9996 & \\
\hline 2.000 & 2.073 & 0.006 & & 0.266 \\
\hline 4.000 & 4.139 & 0.027 & & 0.661 \\
\hline 6.000 & 6.202 & 0.068 & & 1.098 \\
\hline 8.000 & 8.254 & 0.031 & & 0.374 \\
\hline
\end{tabular}

Table 5: The repeatability study (intraday precision) by using UV-spectrophotometry.

\begin{tabular}{|c|c|c|c|c|}
\hline Concentration $(\mathrm{mg} / \mathrm{mL})$ & Average Actual Concentration $(\mathrm{mg} / \mathrm{mL})(\mathrm{n}=3)$ & S.D & $\mathbf{R}^{2}$ For Standard Curve & Precision (\%RSD) \\
\hline 1st Reading & & & 0.9998 & \\
\hline 2.000 & 2.036 & 0.007 & & 0.332 \\
\hline 4.000 & 4.020 & 0.010 & & 0.239 \\
\hline 6.000 & 6.040 & 0.009 & & 0.147 \\
\hline 8.000 & 8.130 & 0.002 & & 0.027 \\
\hline 2nd Reading & & & 0.9998 & \\
\hline 2.000 & 2.043 & 0.002 & & 0.114 \\
\hline 4.000 & 4.043 & 0.004 & & 0.097 \\
\hline 6.000 & 6.056 & 0.017 & & 0.285 \\
\hline 8.000 & 8.062 & 0.008 & & 0.102 \\
\hline 3rd Reading & & & 0.9998 & \\
\hline 2.000 & 2.031 & 0.003 & & 0.159 \\
\hline 4.000 & 4.103 & 0.003 & & 0.068 \\
\hline 6.000 & 6.054 & 0.007 & & 0.108 \\
\hline 8.000 & 8.058 & 0.015 & & 0.183 \\
\hline
\end{tabular}

Table 6: The accuracy of the method was evaluated based on the average recovery and the \%RSD value of each concentration.

\begin{tabular}{ccccc}
\hline Nominal Concentration $(\mathbf{m g} / \mathbf{m L})$ & Actual Concentration $(\mathbf{m g} / \mathbf{m L})(\mathbf{n}=\mathbf{3})$ & Average \% Recovery & S.D & \%RSD \\
\hline 2.000 & 2.009 & 100.448 & 0.847 & 0.844 \\
4.000 & 4.021 & 100.516 & 1.118 & 1.113 \\
6.000 & 6.094 & 101.561 & 1.376 & 1.355 \\
8.000 & 8.056 & 100.701 & 0.120 & 0.119 \\
\hline
\end{tabular}

Table 7: The correlation coefficient $\mathrm{R}^{2}$ values and the linear equations.

\begin{tabular}{ccc}
\hline Condition & Linear Equation & $\mathbf{R}^{2}$ Value \\
\hline $\mathbf{4 2 1} \mathbf{~ n m}$ Wavelength & $y=0.070 x+0.005$ & 0.9991 \\
$\mathbf{4 1 5} \mathbf{~ m}$ Wavelength & $y=0.069+0.009$ & 0.9993 \\
Second Analyst & $y=0.069+0.004$ & 0.9996 \\
\hline
\end{tabular}

\section{Robustness}

The wavelength determined by the UV module was modified with small adjustment and the same concentrations used in constructing the linearity were subjected to the new wavelength. For robustness, the actual wavelength was adjusted to $421 \mathrm{~nm}$ and $415 \mathrm{~nm}$ before the linearity for both wavelengths was determined. From the data in Table 7, it is apparent that small changes done intentionally to the developed method is not affecting the reliability in quantifying the concentration of gentamicin in the given solution. Although the changes were done with the values of $+3 \mathrm{~nm}$ and $-3 \mathrm{~nm}$ from the actual wavelength, $\mathrm{R}^{2}$ values obtained for both adjusted wavelengths were not less than 0.995. Similar observation was acquired with the construction of standard curve by second analyst. The $\mathrm{R}^{2}$ value was 0.9996 and still above acceptance limit. Hence, this proved that the method developed to quantify gentamicin-ninhydrin complex was robust enough to stand small intentional changes.

\section{CONCLUSION}

The quantification method for gentamicin in the form of gentamicin-ninhydrin complex was developed and validated by using UV-spectrophotometry. The basic requirements for the acceptance criteria underlined by ICH Q2 (R1) guidelines where met and fulfilled. The exploration of the relationship between the concentration of gentamicin and the UV absorbance value in order to develop an analytical method for in vitro rapid quantification was successful. Therefore in summary, the current study suggested that this analytical method was rapid, precise, specific, accurate, robust and cost effective to be used in quantifying gentamicin for in-vitro assessment. In addition, the microparticles fabrication 
process was proven to be suitable in encapsulating gentamicin by using PLGA without compromising the efficacy of the antibiotic itself.

\section{REFERENCES}

Šoltés, L. Aminoglycoside antibiotics - two decades of their HPLC bioanalysis. Biomedical Chromatography, 1999; 13(1), 3-10.

Ariano, R. E., Zelenitsky, S. A., and Kassum, D. A. Aminoglycoside-induced vestibular injury: maintaining a sense of balance. Annals of Pharmacotherapy, 2008; 42(9), 1282-1289.

Al-Hamad, A. Improving gentamicin dosing: A suggested approach to a simplified once-daily dosing schedule. Journal of Infection and Public Health, 2014; 7(3), 247-248.

Chung, Y. Y., and Huang, T. W. Microencapsulation of gentamicin in biodegradable PLA and/or PLA/PEG copolymer. Journal of Microencapsulation, 2001; 18(4), 457-465.

Frutos, P., Torrado, S., Perez-Lorenzo, M. E., and Frutos, G. A validated quantitative colorimetric assay for gentamicin. J. Pharm. Biomed. Anal., 2000; 21, 1149-1159.

McLawhon, R. W. 2012. Chapter 10 - Guidelines for the Monitoring of Vancomycin, Aminoglycosides and Certain Antibiotics. In A. Dasgupta (Ed.), Therapeutic Drug Monitoring (pp. 197-218). Boston: Academic Press.

Balmayor, E. R., Baran, E. T., Azevedo, H. S., and Reis, R. L. Injectable biodegradable starch/chitosan delivery system for the sustained release of gentamicin to treat bone infections. Carbohydrate Polymers, 2012; 87(1), 32-39.

Abed, N., and Couvreur, P. Nanocarriers for antibiotics: A promising solution to treat intracellular bacterial infections. International Journal of Antimicrobial Agents, 2014; 43(6), 485-496.

Yoshizawa, S., Fourmy, D., and Puglisi, J. D. Structural origins of gentamicin antibiotic action. The EMBO journal, 1998; 17(22), 64376448.

Ismail, H., Fahmi, A., Doolaanea, A. M., Awang, M., and Mohamed, F. High initial burst release of gentamicin formulated as PLGA microspheres implant for treating orthopaedic infection. International Journal of Pharmacy and Pharmaceutical Sciences, 2012; 4 (supp 4), 685691.

Friess, W., and Schlapp, M. Release mechanisms from gentamicin loaded poly (lactic-co-glycolic acid)(PLGA) microparticles. Journal of Pharmaceutical Sciences, 2002 91(3), 845-855.

Schlapp, M., and Friess, W. Collagen/PLGA microparticle composites for local controlled delivery of gentamicin. Journal of Pharmaceutical Sciences, 2003; 92(11), 2145-2151.

Ismail, A. F. H., Doolaanea, A. A., Mohamed, F., Mansor, N. I., Shafri, M. A. M., and Yusof, F. A. Method Development and Validation using UV Spectrophotometry for Nigella sativa Oil Microparticles Quantification. J App Pharm Sci, 2015; 5(9), 082-088.
Rajesh, T., Kumari, A. S., Reddy, S. R., and Kumar, A. A. UV Spectrophotometric Method Development And Validation For The Quantitative Estimation of Acamprosate Calcium In Tablets. Int J Pharm Pharm Sci, 2014; 6(8), 363-366.

Rajitha, K., Prasanna, N. L., Vasundhara, G., Kumar, R. N., and Kumar, A.A. UV Spectrophotometric Method Development And Validation For The Simultaneous Quantitative Estimation of Mebeverine Hydrochloride And Chlordiazepoxide In Capsules. Int J Pharm Pharm Sci, $2011 ; 6(8), 345-349$.

Iqbal, M., Alam, P., and Anwer, M. T. High Performance Liquid Chromatographic Method with Fluorescence Detection for the Estimation of Thymoquinone in Nigella sativa Extracts and Marketed Formulations. Open Access Scientific Reports, 2013; 2(2), 1-6.

Andrews, J. M. Determination of minimum inhibitory concentrations. Journal of Antimicrobial Chemotherapy, 2001; 48(suppl 1), 5-16.

Behera, S., Ghanty, S., Ahmad, F., Santra, S., and Banerjee, S. UV-Visible Spectrophotometric Method Development and Validation of Assay of Paracetamol Tablet Formulation. Anal Bioanal Techniques, 2012; 3(6), 1-6.

Lecároz, C., Campanero, M. A., Gamazo, C., and BlancoPrieto, M. J. Determination of gentamicin in different matrices by a new sensitive high-performance liquid chromatography-mass spectrometric method. Journal of Antimicrobial Chemotherapy, 2006; 58(3), 557-563.

Yusof, F.A., Shafri, M. A. M., Yaakob, K. I., Yusof, N. M., and Mohamed, F. Formulation and stability testing of Gentamicin-N. sativa fusion emulsions for osteo-healing application. International Journal of Pharmacy and Pharmaceutical Sciences, 2014; 6(11).

\section{How to cite this article:}

Ismail AFH, Mohamed F, Rosli LMM, Shafri MAM, Haris MS, Adina AB. Spectrophotometric Determination of Gentamicin Loaded PLGA Microparticles and Method Validation via Ninhydrin-Gentamicin Complex as a Rapid Quantification Approach. J App Pharm Sci, 2016; 6 (01): 007-014. 